\section{Super-Eddington Mechanical Power of an Accreting Black Hole in M83}

\author{
R. Soria, ${ }^{1 *}$ K. S. Long, ${ }^{2}$ W. P. Blair, ${ }^{2,3}$ L. Godfrey, ${ }^{4}$ K. D. Kuntz, ${ }^{3,5}$ E. Lenc, ${ }^{6,7}$ \\ C. Stockdale, ${ }^{8}$ P. F. Winkler ${ }^{9}$
}

Mass accretion onto black holes releases energy in the form of radiation and outflows. Although the radiative flux cannot substantially exceed the Eddington limit, at which the outgoing radiation pressure impedes the inflow of matter, it remains unclear whether the kinetic energy flux is bounded by this same limit. Here, we present the detection of a radio-optical structure, powered by outflows from a non-nuclear black hole. Its accretion disk properties indicate that this black hole is less than 100 solar masses. The optical-infrared line emission implies an average kinetic power of $3 \times 10^{40} \mathrm{erg} \mathrm{second}^{-1}$, higher than the Eddington luminosity of the black hole. These results demonstrate kinetic power exceeding the Eddington limit over a sustained period, which implies greater ability to influence the evolution of the black hole's environment.

$\mathrm{F}$ or an accreting black hole $(\mathrm{BH})$ of mass $M$, the Eddington luminosity $\left[L_{\mathrm{Edd}} \approx 1.3 \times\right.$ $10^{38}\left(M / M_{\odot}\right)$ erg s${ }^{-1}$; where $M_{\odot}$ is solar mass] is the maximum photon power achievable for spherically symmetric, radiatively efficient accretion, and the Eddington accretion rate is the

${ }^{1}$ International Centre for Radio Astronomy Research, Curtin University, G.P.O. Box U1987, Perth, WA 6845, Australia. ${ }^{2}$ Space Telescope Science Institute, 3700 San Martin Drive, Baltimore, MD 21218, USA. ${ }^{3}$ Department of Physics and Astronomy, Johns Hopkins University, 3400 North Charles Street, Baltimore, MD 21218, USA. ${ }^{4}$ Netherlands Institute for Radio Astronomy (ASTRON), P.O. Box 2, 7990 AA Dwingeloo, Netherlands. ${ }^{5}$ NASA Goddard Space Flight Center, 8800 Greenbelt Road, Greenbelt, MD 20771, USA. ${ }^{6}$ Sydney Institute for Astronomy, School of Physics, The University of Sydney, New South Wales 2006, Australia. ${ }^{7}$ ARC Centre of Excellence for All-sky Astrophysics (CAASTRO), Sydney, Australia. ${ }^{8}$ Department of Physics, Marquette University, P.O. Box 1881, Milwaukee, WI 53201-1881, USA. ${ }^{9}$ Department of Physics, Middlebury College, Middlebury, VT 05753, USA.

*Corresponding author. E-mail: roberto.soria@icrar.org corresponding mass accretion rate. However, BHs can also release energy through mechanical channels (kinetic energy of jets and winds). The balance between radiative and kinetic output depends partly on the mass accretion rate. For accretion rates $\sim 0.03$ to 0.5 times the Eddington rate, the $\mathrm{BH}$ power output is entirely dominated by thermal disk emission without a jet (1-3), whereas for rates approaching or exceeding the Eddington rate, we expect radiatively driven outflows, possibly coexisting with faster, collimated jets $(4,5)$.

There is circumstantial evidence that nonisotropic photon luminosity can mildly exceed the Eddington limit (3,6-8), but can BHs with supercritical accretion have powerful jets, and can the mechanical power $P_{\mathrm{J}}$ exceed $L_{\mathrm{Edd}}$ for an extended period of time? Empirical evidence of super-Eddington jet power was presented for a sample of radio-loud quasars $(9,10)$. However, ambiguities in the $\mathrm{BH}$ masses and in the conversion from 151-MHz radio flux to jet power
(11-15) make these results uncertain. In the local universe, shock-ionized gas around ultraluminous $\mathrm{X}$-ray sources (ULX bubbles) provides strong evidence of sustained and powerful mechanical output from sources that are thought to have high accretion rates (16-22). Typical ULX bubbles have sizes of $\sim 50$ to $300 \mathrm{pc}$ and require a mechanical input power of $\sim 10^{39}$ to $10^{40} \mathrm{erg} \mathrm{s}^{-1}$ for $\sim 10^{5}$ years. However, there are no BH mass measurements for such ULXs: If they are powered by $\sim 10 M_{\odot}$ BHs, their radiative and/or mechanical power would, in some cases, exceed the Eddington value by a factor of 10 , but if they are powered by BHs of mass $>100 M_{\odot}$ (known as intermediate-mass BHs), they may not exceed the Eddington limit. In the Milky Way, the most energetic BH bubble is probably SS 433/W50, with an average mechanical power of $\sim 10^{39} \mathrm{erg} \mathrm{s}^{-1}$ over an age of a few $10^{4}$ years $(23-28)$.

Here, we report the detection of a powerful microquasar in the inner disk of the spiral galaxy M83 (NGC 5236), located at a distance of $4.61 \pm 0.26 \mathrm{Mpc}(29)$. We are conducting a multiwavelength study of $\mathrm{x}$-ray source populations in M83. Our observations include 5.5- and 9.0-GHz images taken with the Australia Telescope Compact Array (ATCA) on 28 to 30 April 2011, for a total observation time of 36 hours. We also used archival data of M83 taken with the Australian Long Baseline Array (LBA) on 13 May 2005, for a total of 9 hours, at $2.3 \mathrm{GHz}(30)$. In the infrared (IR)-optical bands, we used archival Hubble Space Telescope/Wide Field Camera 3 (HST/WFC3) images of the inner disk of the galaxy in several broad- and narrow-band filters (data taken on 20 to 26 August 2009). We observed M83 with the Chandra X-ray Observatory/ACIS-S camera 10 times between December 2010 and December 2011, as part of a large program to study $\mathrm{x}$-ray source populations of a star-forming galaxy; the total exposure time was $729 \mathrm{ks}$. In addition, we used an archival 50-ks x-ray observation of M83
Fig. 1. Location of MQ1 in the inner region of the $M 83$ galaxy. (A) HST/WFC3 image of the central region of $\mathrm{M} 83$ in the $\mathrm{F} 657 \mathrm{~N}$ filter, with superposed $9.0-\mathrm{GHz}$ surface brightness contours, from our ATCA data. Contour levels are $0.10,0.18$, $0.40,0.78,1.32$, and $2.0 \mathrm{~m}$ ]y per beam. The beam size is $2^{\prime \prime} .3 \times 0^{\prime \prime} .9$ at a position angle of $173^{\circ}$. The root mean square noise level in source-free regions of the map is $0.025 \mathrm{~m}$ jy per beam, and the peak intensity of MQ1 is 0.9 mJy per beam, but this includes a small contribution from the surrounding extended emission. Labels mark the location of the microquasar MQ1 and of a nearby SNR used for astrometric alignment. The blue circle (radius $0^{\prime \prime} .6$ ) marks the nucleus of M83. (B) Chandra/ACIS x-ray color image of the same field (red, 0.35 to 1.1 keV; green, 1.1 to $2.6 \mathrm{keV}$; blue, 2.6 to $8.0 \mathrm{keV}$ ).
A

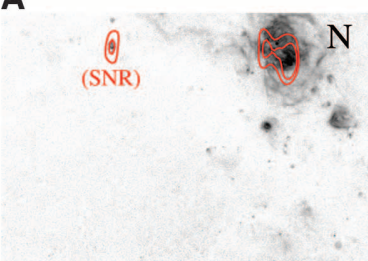

$\mathrm{E}$

$10^{\prime \prime}=220 \mathrm{pc}$
B

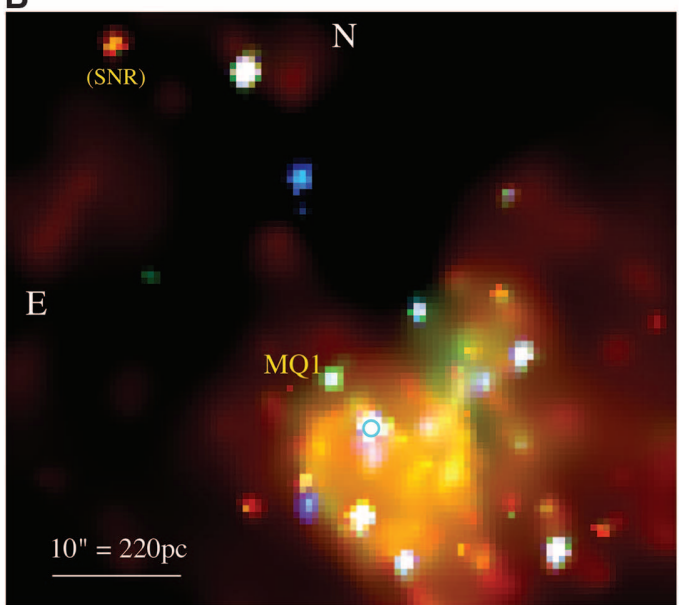




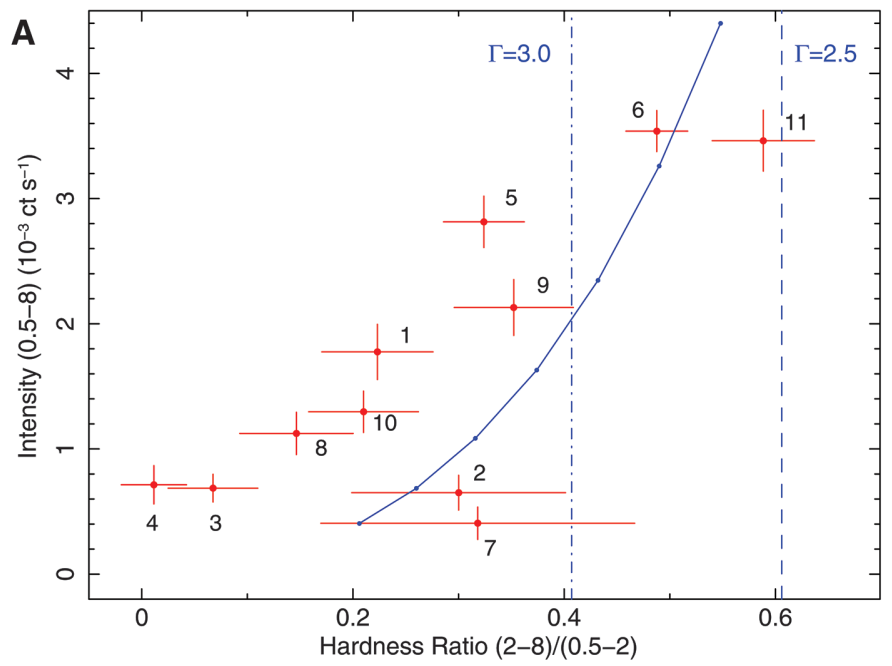

Fig. 2. X-ray hardness and spectral properties. (A) X-ray hardness-versusintensity diagram of MQ1 from our sequence of Chandra observations. The number next to each data point indicates the order in which the observations were taken (table S1). The blue line denotes the expected location of a diskblackbody spectrum with temperature $k_{\mathrm{B}} T_{\text {in }}$ varying (from top to bottom) from 0.80 to $0.50 \mathrm{keV}$ in steps of $0.05 \mathrm{keV}$. Dashed and dash-dotted lines mark the

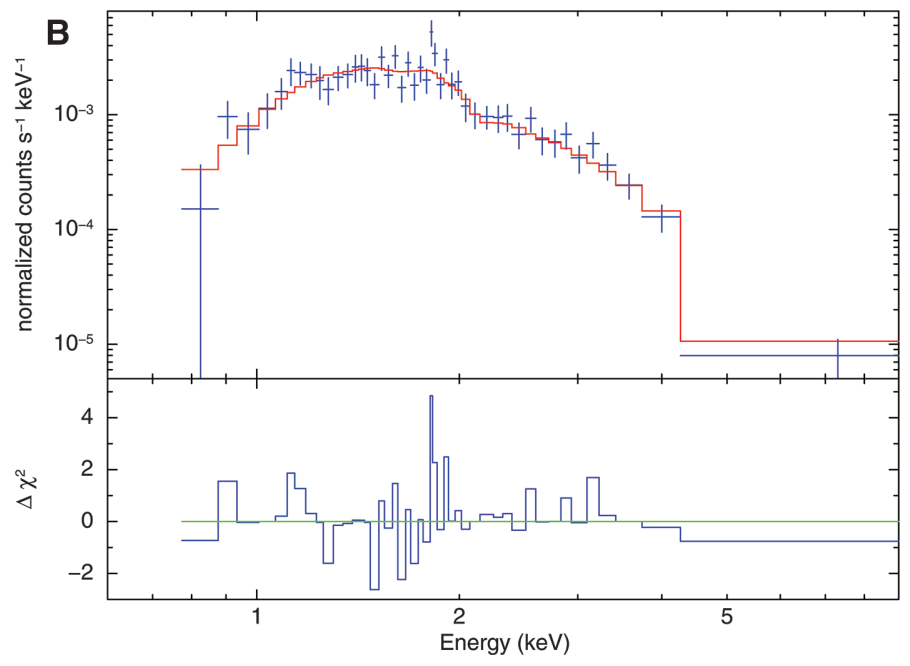

expected hardness of sources with the same absorbing column and power-law photon indices $\Gamma=2.5$ and 3, respectively: The observed values are clearly inconsistent with the low/hard state $(\Gamma<2)$ at all epochs. $c s^{-1}$, counts per second. (B) Combined x-ray spectrum from the Chandra ObsID 12994 (6th observation) and 14342 (11th observation), when the source was brightest. The spectrum is well fitted with an absorbed disk-blackbody model, with parameters typical of stellar-mass BHs (table S3).
A

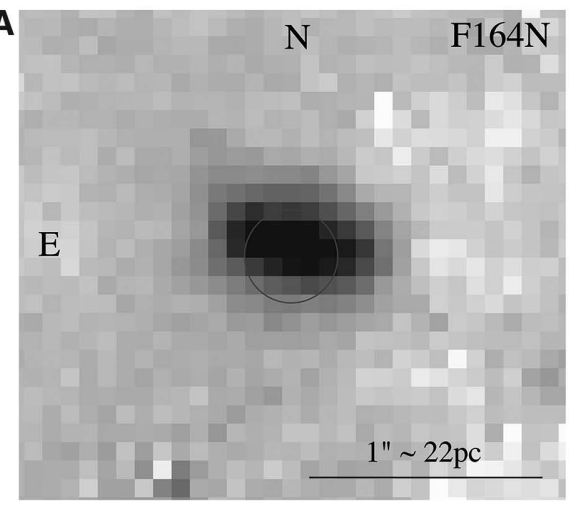

C

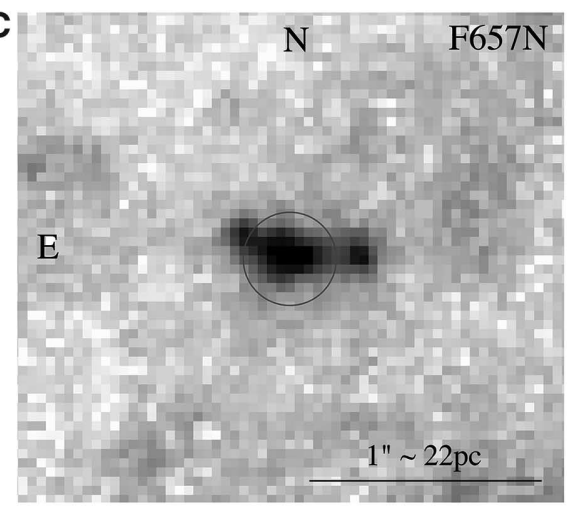

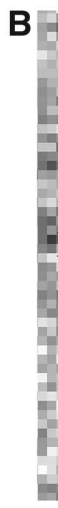

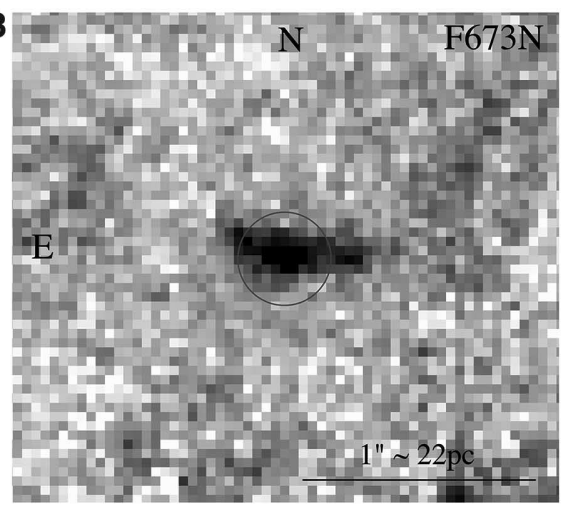

D

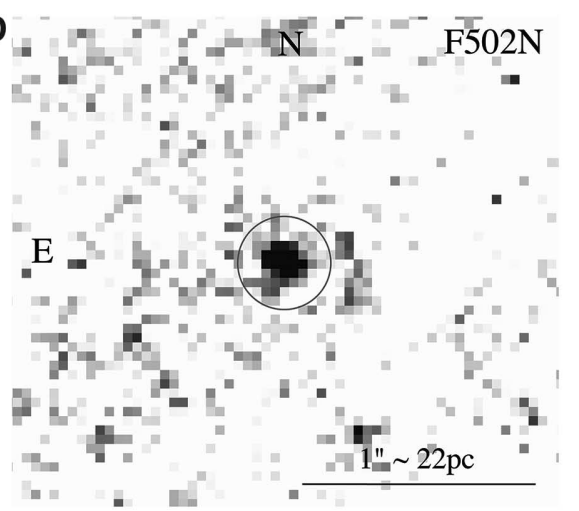

Fig. 3. Optical-IR line emission. (A to D) HST/WFC3 image of the microquasar in four continuumsubtracted bands. Clockwise from top left: [Fell] $\lambda=1.644 \mu \mathrm{m}$; [SII] $\lambda \lambda 6716,6731$; [OIII] $\lambda 5007$; and $\mathrm{H} \alpha+[\mathrm{NII}] \lambda \lambda 6548,6584$. The blue circle indicates the centroid of the ATCA/Chandra source and has a radius of $0^{\prime \prime} .2$.

obtained with Chandra/ACIS-S in April 2000 $(31,32)$.

Our ATCA images show a luminous radio source at right ascension $(\mathrm{RA})=13$ hours $37 \mathrm{~min}$
$1.123 \mathrm{~s}( \pm 0.005 \mathrm{~s})$, Dec. $=-29^{\circ} 51^{\prime} 52^{\prime \prime} .17\left( \pm 0^{\prime \prime} .10\right)$ (J2000), $5^{\prime \prime}$ northeast of the optical nucleus of M83 and just outside the intensely star-forming region that surrounds it (Fig. 1 and fig. S1). It has a flux density $S_{5.5}=1.4 \pm 0.1$ millijansky (mJy) and $S_{9.0}=0.80 \pm 0.05 \mathrm{mJy}$ at a frequency $(\mathrm{v})$ of 5.5 and $9.0 \mathrm{GHz}$, respectively, which corresponds to a radio luminosity $\approx 2 \times 10^{35} \mathrm{erg} \mathrm{s}^{-1}$, similar to those of the two most powerful microquasar bubbles known to date, IC $342 \mathrm{X}-1$ and NGC $7793-\mathrm{S} 26(19,20,22)$. At $5 \mathrm{GHz}$, this radio source is $\approx 30$ times more radio-luminous than SS 433/W50 (24, 26, 27). Henceforth, we identify this source as MQ1. Its steep spectral index $\alpha \approx$ $1.1\left(S_{v} \sim v^{-\alpha}\right)$ implies optically thin synchrotron emission, possibly from an aging population of electrons. MQ1 is unresolved in the ATCA maps, implying a diameter $\lesssim 1^{\prime \prime} \approx 22 \mathrm{pc}$. In the LBA map, an unresolved source (size $\lesssim 2 \mathrm{pc}$ ) is detected at $\mathrm{RA}=13$ hours $37 \mathrm{~min} 1.136 \mathrm{~s}( \pm 0.003 \mathrm{~s})$, Dec. $=-29^{\circ} 51^{\prime} 52^{\prime \prime} .15\left( \pm 0^{\prime \prime} .02\right)(\mathrm{J} 2000)$ with a flux density of $S_{2.3}=0.22 \pm 0.04 \mathrm{mJy}$ at $2.3 \mathrm{GHz}$, a factor of $\approx 10$ to 15 times less than the total expected flux density at that frequency, based on the ATCA data. This indicates that $\sim 90 \%$ of the emission is extended over a region of at least a few parsecs in diameter (much larger than the LBA beam but smaller than the ATCA beam). The LBA source is offset to the east of the ATCA centroid by $\approx 0^{\prime \prime} .2 \pm 0 " .1$ (fig. S2).

We interpret the radio source as emission from a radio bubble or radio lobes (that is, from an expanding structure of shocked plasma) inflated by the mechanical power of the BH; synchrotronemitting electrons are produced where the jet heads hit the interstellar medium (ISM). Similar structures have been found in other non-nuclear BHs: notably, the ring around Cyg X-1 (33), the "ears" of SS 433 (34), and the bubbles around several ULXs (35). We speculate that the LBA radio source arises from the eastern hot spot, where the head of a jet strikes the ISM. The 
nondetection of a compact core in the LBA (flux density $S_{2.3} \lesssim 0.1 \mathrm{mJy}$ per beam) does not imply that the jet is currently turned off, as we estimate [scaling from the power and luminosity of galactic microquasars, e.g., (36)] that the radio flux density of the MQ1 core would always be less than or equivalent to a few microjansky, even for an efficient jet production at high accretion rates.

Our Chandra study shows a luminous pointlike source, coincident with MQ1 to within the $\mathrm{X}$-ray positional uncertainty of $0^{\prime \prime} .3$, whose characteristics provide an estimate of the $\mathrm{BH}$ mass. The x-ray flux and hardness vary from one observation to another (Fig. 2 and fig. S3) and also within observations (fig. S4). This variability rules out emission from shocked gas; the source must be an accreting compact object. The x-ray spectrum is always consistent with thermal disk emission (2); it is never consistent with the power-lawdominated low/hard state. When the source is brightest [Chandra X-ray Observatory identification number (ObsID) 12994 and ObsID 14342], a standard disk-blackbody model fit [tbabs $\times$ $t b a b s \times$ diskbb in XSPEC version 12.6 .0 (37)] gives a characteristic peak temperature $k_{\mathrm{B}} T_{\text {in }} \approx$ $0.7 \mathrm{keV}$ (where $k_{\mathrm{B}}$ is the Boltzmann constant), apparent inner-disk radius $r_{\text {in }}(\cos \theta)^{1 / 2} \approx 50 \mathrm{~km}$ (where $\theta$ is the viewing angle: face-on for $\theta=0$ ), and unabsorbed 0.5 - to $8-\mathrm{keV}$ luminosity $\approx 7.4 \times$ $10^{37}(\cos \theta)^{-1} \mathrm{erg} \mathrm{s}^{-1}$. The inferred BH mass depends on the viewing angle and the $\mathrm{BH}$ spin: $M \approx$ $\left(1.19 c^{2} / G\right)(1 / \alpha) r_{\text {in }}$ (here, $c$ is the speed of light and $G$ is the gravitational constant) (38), where $\alpha \equiv\left(c^{2} / G\right)\left(R_{\mathrm{isco}} / M\right)=6$ for a nonrotating $\mathrm{BH}$ and $1.24<\alpha<6$ for astrophysically plausible spinning $\mathrm{BHs}(39,40)$, and $R_{\text {isco }}$ is the innermost stable circular orbit. From our standard diskblackbody fit, we obtain $M \approx\left(42.6_{-9.3}^{+12.8}\right)(1 / \alpha)$ $(\cos \theta)^{-1 / 2} M_{\odot}$. Thus, for arbitrary midrange values ( $\cos \theta \sim 0.5$, Kerr parameter $a \sim 0.5$, implying $\alpha \sim 4$ ), the spectral model, fitted temperature, inner-disk radius, and luminosity are similar to those found in galactic stellar-mass BHs $\left(M \sim 10 M_{\odot}\right)$ accreting at $\sim 10 \%$ of the Eddington rate, which supports the stellar-mass $\mathrm{BH}$ identification for MQ1 (Occam's razor). For the most extreme (less likely) choice of parameters in their physically acceptable range $\left(\theta=85^{\circ}, a \approx 0.998\right)$, the bestfitting $\mathrm{BH}$ mass estimate is $M \approx 115 M_{\odot}$.

There is an optical emission line source at the position of MQ1 that was previously identified with HST (41) and classified as a supernova remnant (SNR) within the nuclear region. Closer inspection of the optical source in the continuumsubtracted $\mathrm{H} \alpha+[\mathrm{NII}] \lambda \lambda 6548,6584,[\mathrm{FeII}]$ $\lambda 1.644 \mu \mathrm{m}$, and [SII] $\lambda \lambda 6716,6731 \AA$ images (Fig. 3) shows that it is resolved into a stronger central source with two knots or lobes at either side (a structure reminiscent of SS 433/W50). The sum of the projected distances between the side knots and the central peak is $\approx 0^{\prime \prime} .6 \approx 13 \mathrm{pc}$. There are no other bright stars or sources of optical continuum emission at this location (fig. S5); thus, the extended optical source is a physical structure rather than a random alignment of three or more unrelated emission line objects. We used nearby sources detected in more than one band to refine the relative astrometry of the HST image with respect to the radio coordinates, and we conclude that the optical core, the ATCA centroid, and the $\mathrm{x}$-ray position are coincident within $\approx 0^{\prime \prime} .15$. The eastern knot of $\mathrm{H} \alpha$ emission may be associated with the compact LBA source (fig. S2). We suggest that the optical structure represents the core and hot spots and/or lobes of the microquasar.

Optical line emission from the shocked gas is a reliable proxy for mechanical power. For a fully radiative shock expanding into the ISM with velocity $v_{\mathrm{s}}$, the total radiative flux equals the flux of mechanical energy through the shock $(35,42)$. Standard bubble theory (43) shows that the bolometric photon luminosity $L_{\mathrm{T}}$ is related to the long-termaveraged mechanical power as $L_{\mathrm{T}}=(27 / 77) P_{\mathrm{J}}$, with the rest of the power going into the kinetic energy of the expanding shell of swept-up gas and into the thermal energy of the shocked gas between the reverse shock and the swept-up shell. The fraction of bolometric radiative luminosity emitted in a given line (including both the shock and the precursor contributions) can be computed from shock- and photoionization codes such as Modelling And Prediction in Photo-Ionized Nebulae and Gasdynamic Shocks III (MAPPINGS III) (44).

$\mathrm{H} \beta$ or $\mathrm{H} \alpha$ are the lines most often used to estimate microquasar jet power (19). However, in this case both lines (especially $\mathrm{H} \beta$ ) are affected by high optical extinction; the Ho flux from HST imagery includes an uncertain but substantial contribution from [NII] $\lambda \lambda 6548,6584$; and part of the Balmer emission may come from x-ray photoionization due to the central source. Thus, we used instead the flux in the IR line $[\mathrm{FeII}] \lambda=1.644 \mu \mathrm{m}$ as a proxy for the mechanical power. The [FeII] line is a powerful diagnostic tool for shock-heated gas in high-density regions (45-48).

We measured the [FeII] line flux (table S2) from the HST/WFC3 IR-camera image in the F164N filter after subtracting the continuum emission using the F160W image. The apparent (not corrected for extinction) [FeII] luminosity is $\approx 1.1 \times 10^{37} \mathrm{erg} \mathrm{s}^{-1}$, one order of magnitude greater than that observed from SNRs in the spiral arms. We adopted an extinction $A_{V} \approx 3.9$ magnitude (mag), as inferred from the x-ray column density $\left[n_{\mathrm{H}}=\left(8.6_{-1.5}^{+1.8}\right) \times 10^{21} \mathrm{~cm}^{-2}\right]$ using the conversion relation of (49), with a likely uncertainty range $A_{V} \sim 3.8$ to 5 mag. Taking into account the corresponding IR extinction at $1.64 \mu \mathrm{m}$ $\left(A_{1.64 \mu \mathrm{m}}\right) \approx 0.70 \mathrm{mag}$, the emitted luminosity $L_{[\mathrm{FeII}]}=(2.1 \pm 0.2) \times 10^{37} \mathrm{erg} \mathrm{s}^{-1}$ (table S2).

To estimate the mechanical power, we assume a preshock atomic number density $n_{0} \sim 100 \mathrm{~cm}^{-3}$, typical of the inner disk-nuclear region of M83 $(41,50,51)$. Then, from MAPPINGS III models (44), and assuming equipartition between magnetic and gas pressure, we derive that $L_{[\mathrm{FeII}]}=$ $(0.7 \pm 0.1) \times 10^{-3} P_{\mathrm{J}}$ over a wide range of plausible shock velocities (fig. S6). We conclude that $P_{\mathrm{J}} \approx 3 \times 10^{40} \mathrm{erg} \mathrm{s}^{-1}>L_{\mathrm{Edd}}$ (from the $\mathrm{BH}$ mass constraints). We can derive other physical parameters using a well-known self-similar expansion solution for jet-driven bubbles and lobes $(43,52)$. The characteristic radius $R$ (distance between core and hot spots) scales as $R \approx 0.76 P_{\mathrm{J}}^{1 / 5} t^{3 / 5} \rho_{0}{ }^{-1 / 5}$ where $\rho_{0} \approx \mu m_{\mathrm{p}} n_{0}\left(m_{\mathrm{p}}\right.$ is the proton mass and $\mu$ the mean atomic weight). Taking $P_{\mathrm{J}} \approx 3 \times$ $10^{40} \mathrm{erg} \mathrm{s}^{-1}, R \approx 0 " .3 \approx 6.7 \mathrm{pc}$ (neglecting projection effects), and $\mu=1.38$, we infer a characteristic expansion age $t \approx 4.9 \times 10^{11} \mathrm{~s} \approx 16,000$ years. The expansion velocity $v_{\mathrm{s}}=\mathrm{d} R / \mathrm{d} t=(3 / 5) R / t \approx$ $250 \mathrm{~km} \mathrm{~s}^{-1}$. Taking $n_{0} \sim 50$ to $200 \mathrm{~cm}^{-3}$ does not substantially change the power but gives an age range $\sim 13,000$ to 20,000 years and a velocity range $v_{\mathrm{s}} \sim 200$ to $310 \mathrm{~km} \mathrm{~s}^{-1}$. Mechanical power and shock velocity are similar to those measured in the powerful microquasar NGC $7793 \mathrm{~S} 26(19,20,53)$, but the age of MQ1 is a factor of 10 younger and the ISM density two orders of magnitude higher.

Both the optical-IR and the radio luminosity come from the bubble and/or lobes; therefore, they trace the time-averaged mechanical power. The x-ray luminosity traces the current accretion rate. It is likely that during the Chandra observations, the system had a sub-Eddington luminosity and a weak jet or none at all. It is also likely that in the past, the system had super-Eddington luminosity and jet power: It would have looked like ULXs such as Holmberg IX X-1 or NGC $1313 \mathrm{X}-2$ (luminosity $L_{\mathrm{X}} \approx 2 \times 10^{40} \mathrm{erg} \mathrm{s}^{-1}$ ), surrounded by ionized bubbles with mechanical power $P_{\mathrm{J}} \sim 10^{40} \mathrm{erg} \mathrm{s}^{-1}$ (35). If the x-ray core switches off or fades, the surrounding radio-optical bubble remains bright.

For MQ1, we have been able to constrain the $\mathrm{BH}$ mass from $\mathrm{x}$-ray spectral analysis because the $\mathrm{BH}$ is currently in a disk-dominated state. None of the other microquasars with similar mechanical power has a reliable constraint on its $\mathrm{BH}$ mass, because none of them has been observed in this state. Combined with an accurate determination of time-averaged mechanical power from shock-ionized line luminosities, this enables a robust estimation of the (mechanical) Eddington ratio. Thus, we have identified a system that has had (on average) a super-Eddington mechanical power for $\sim 2 \times 10^{4}$ years (most likely, a few times above Eddington). MQ1 provides empirical evidence that BHs can sustain super-Eddington mechanical power for an extended period of time. During those phases, BHs inject more energy into the surrounding medium than would be inferred on the basis of their Eddington limits. The existence of super-Eddington mechanical power is important for the modelling of jet production mechanisms, as well as the evolution of fastgrowing supermassive BHs in the early universe and their effect on their host galaxies.

References and Notes

1. J. E. McClintock, R. A. Remillard, in Compact Stellar $X$-ray Sources, W. Lewin, M. van der Klis, Eds. (Cambridge Astrophysics Series, no. 39, Cambridge Univ. Press, Cambridge, 2009), p. 157. 
2. R. A. Remillard, ]. E. McClintock, Annu. Rev. Astron. Astrophys. 44, 49-92 (2006).

3. N. I. Shakura, R. A. Sunyaev, Astron. Astrophys. 24, 337-355 (1973).

4. K. Ohsuga, S. Mineshige, Astrophys. J. 736, 20 (2011)

5. A. R. King, K. A. Pounds, Mon. Not. R. Astron. Soc. 345 , 657-659 (2003)

6. M. C. Begelman, Astrophys. J. 643, 1065-1080 (2006)

7. J. Poutanen, G. Lipunova, S. Fabrika, A. G. Butkevich, P. Abolmasov, Mon. Not. R. Astron. Soc. 377, 1187-1194 (2007).

8. A. D. Sutton, T. P. Roberts, M. J. Middleton, Mon. Not. R. Astron. Soc. 435, 1758-1775 (2013).

9. B. Punsly, Mon. Not. R. Astron. Soc. 374, L10-L14 (2007).

10. B. Punsly, Astrophys. J. 728, L17 (2011).

11. C. J. Willott, S. Rawlings, K. M. Blundell, M. Lacy, Mon. Not. R. Astron. Soc. 309, 1017-1033 (1999)

12. K. M. Blundell, S. Rawlings, Astron. J. 119, 1111-1122 (2000).

13. B. Punsly, Astrophys. J. 623, L9-L12 (2005).

14. L. E. H. Godfrey, S. S. Shabala, Astrophys. J. 767, 12 (2013).

15. S. S. Shabala, L. E. H. Godfrey, Astrophys. J. 769, 129 (2013).

16. M. W. Pakull, L. Mirioni, "Optical counterparts of ultraluminous x-ray sources," in Proceedings of the Symposium "New Visions of the X-ray Universe in the XMM-Newton and Chandra Era," 26 to 30 November 2001 (ESTEC, The Netherlands, 2002); http://arxiv.org/abs/astro-ph/0202488.

17. M. W. Pakull, L. Mirioni, Rev. Mex. Astron. Astrophys. Conf. Series 15, 197-199 (2003)

18. M. W. Pakull, F. Grisé, in Proceedings of A Population Explosion. The Nature \& Evolution of X-ray Binaries in Diverse Environments, R. M. Bandyopadhyay, S. Wachter, D. Gelino, C. R. Gelino, Eds. (American Institute of Physics, New York, 2008), vol. 1010, p. 303.

19. M. W. Pakull, R. Soria, C. Motch, Nature 466, 209-212 (2010).

20. R. Soria, M. W. Pakull, J. W. Broderick, S. Corbel, C. Motch, Mon. Not. R. Astron. Soc. 409, 541-551 (2010).

21. H. Feng, R. Soria, New Astron. Rev. 55, 166-183 (2011)
22. D. Cseh et al., Astrophys. J. 749, 17 (2012).

23. Yu. M. Krivosheyev, G. S. Bisnovatyi-Kogan, A. M. Cherepashchuk, K. A. Postnov, Mon. Not. R. Astron. Soc. 394, 1674-1684 (2009).

24. S. Fabrika, Astrophys. Space Phys. Rev. 12, 1-152 (2004).

25. H. L. Marshall, C. R. Canizares, N. S. Schulz, Astrophys. J. 564, 941-952 (2002).

26. G. M. Dubner, M. Holdaway, W. M. Goss, I. F. Mirabel, Astron. J. 116, 1842-1855 (1998)

27. B. Margon, Annu. Rev. Astron. Astrophys. 22, 507-536 (1984).

28. W. J. Zealey, M. A. Dopita, D. F. Malin, Mon. Not. R. Astron. Soc. 192, 731-743 (1980).

29. A. Saha, F. Thim, G. A. Tammann, B. Reindl, A. Sandage, Astrophys. J. 165 (suppl.), 108-137 (2006).

30. E. Lenc, thesis, Swinburne University Of Technology, Melbourne, Australia (2009); http://arxiv.org/abs/1401.4791.

31. R. Soria, K. Wu, Astron. Astrophys. 384, 99-111 (2002).

32. R. Soria, K. Wu, Astron. Astrophys. 410, 53-74 (2003)

33. E. Gallo et al., Nature 436, 819-821 (2005).

34. R. Elston, S. Baum, Astron. J. 94, 1633-1640 (1987).

35. M. W. Pakull, F. Grisé, C. Motch, in Proceedings of the International Astronomical Union Symposium 230, E. J. A. Meurs, G. Fabbiano, Eds. (Cambridge Univ. Press, Cambridge, 2006), p. 293.

36. S. Corbel et al., Mon. Not. R. Astron. Soc. 428 2500-2515 (2013).

37. K. A. Arnaud, ASP Conf. Ser. 101, 17 (1996).

38. A. Kubota et al., Publ. Astron. Soc. Jpn. 50, 667-673 (1998).

39. J. M. Bardeen, W. H. Press, S. A. Teukolsky, Astrophys. J. 178, 347-370 (1972).

40. K. S. Thorne, Astrophys. J. 191, 507-520 (1974).

41. M. A. Dopita et al., Astrophys. J. 710, 964-978 (2010).

42. M. A. Dopita, R. S. Sutherland, Astrophys. J. 102 (suppl.), 161 (1996).

43. R. Weaver, R. McCray, ]. Castor, P. Shapiro, R. Moore, Astrophys. J. 218, 377 (1977).

44. M. G. Allen, B. A. Groves, M. A. Dopita, R. S. Sutherland, L. J. Kewley, Astrophys. J. 178 (suppl.), 20-55 (2008).

45. E. Oliva, A. F. M. Moorwood, I. J. Danziger, Astron. Astrophys. 214, 307-320 (1989).
46. T. Morel, R. Doyon, N. St-Louis, Mon. Not. R. Astron. Soc. 329, 398-410 (2002)

47. M. Reiter, N. Smith, Mon. Not. R. Astron. Soc. 433, 2226-2239 (2013)

48. ].-H. Shinn et al., Astrophys. ]. 777, 45 (2013).

49. T. Güver, F. Özel, Mon. Not. R. Astron. Soc. 400, 2050-2053 (2009).

50. W. P. Blair, P. F. Winkler, K. S. Long, Astrophys. J. 203 (suppl.), 8 (2012).

51. W. P. Blair, P. F. Winkler, K. S. Long, Astrophys. J. 207 (suppl.), 40 (2013).

52. C. R. Kaiser, P. A. Alexander, Mon. Not. R. Astron. Soc. 286, 215-222 (1997)

53. M. A. Dopita, J. L. Payne, M. D. Filipović, T. G. Pannuti, Mon. Not. R. Astron. Soc. 427, 956-967 (2012).

Acknowledgments: We thank M. W. Pakull for his insightful lessons and comments on ULX bubbles, J. Miller-Jones and A. Moin for their help with the ATCA observations and data analysis, and P. P. Plucinsky for his helpful role in the Chandra study of M83. R.S. acknowledges an Australian Research Council's Discovery Projects funding scheme (project number DP 120102393). W.P.B. acknowledges Space Telescope Science Institute grant G0-12513-01 to Johns Hopkins University. C.S. acknowledges the NASA Wisconsin Space Grant Consortium research grant. P.F.W. acknowledges financial support from the NSF through grant AST-0908566. This work was supported by NASA through Chandra grant G01-12115, issued by the Chandra X-ray Observatory Center, which is operated by the Smithsonian Astrophysical Observatory for and on behalf of NASA under contract NAS8-03060. The Centre for All-sky Astrophysics (CAASTRO) is an Australian Research Council Centre of Excellence, funded by grant CE110001020.

\section{Supplementary Materials}

www.sciencemag.org/content/343/6177/1330/suppl/DC1

Supplementary Text

Figs. S1 to $\mathrm{S} 6$

Tables $\mathrm{S} 1$ to $\mathrm{S} 5$

References (54-84)

20 November 2013; accepted 12 February 2014

Published online 27 February 2014;

10.1126/science.1248759

\section{Large-Amplitude Spin Dynamics} Driven by a THz Pulse in Resonance with an Electromagnon

T. Kubacka, ${ }^{1 *}$ ]. A. Johnson, ${ }^{2}$ M. C. Hoffmann, ${ }^{3}$ C. Vicario, ${ }^{4}$ S. de Jong, ${ }^{3}$ P. Beaud, ${ }^{2}$ S. Grübel, ${ }^{2}$ S.-W. Huang, ${ }^{2}$ L. Huber, ${ }^{1}$ L. Patthey, ${ }^{4}$ Y.-D. Chuang, ${ }^{5}$ J. J. Turner, $^{3}$ G. L. Dakovski, ${ }^{3}$ W.-S. Lee, ${ }^{3}$ M. P. Minitti, ${ }^{3}$ W. Schlotter, ${ }^{3}$ R. G. Moore, ${ }^{6}$ C. P. Hauri, ${ }^{4,7}$ S. M. Koohpayeh, ${ }^{8}$ V. Scagnoli, ${ }^{2}$ G. Ingold, ${ }^{2}$ S. L. Johnson, ${ }^{1} \dagger$ U. Staub ${ }^{2} \dagger$

Multiferroics have attracted strong interest for potential applications where electric fields control magnetic order. The ultimate speed of control via magnetoelectric coupling, however, remains largely unexplored. Here, we report an experiment in which we drove spin dynamics in multiferroic $\mathrm{TbMnO}_{3}$ with an intense few-cycle terahertz ( $\mathrm{THz}$ ) light pulse tuned to resonance with an electromagnon, an electric-dipole active spin excitation. We observed the resulting spin motion using time-resolved resonant soft x-ray diffraction. Our results show that it is possible to directly manipulate atomic-scale magnetic structures with the electric field of light on a sub-picosecond time scale.

$\mathrm{D}$ ata storage devices based on ferromagnetic or ferroelectric materials depend strongly on domain reorientation, a process that typically occurs over time scales of several nanoseconds. Faster reorientation dynamics may be achievable by using intense electromagnetic (EM) pulses (1). The EM pulses can couple to magnetism either indirectly via electronic excitations (2) or directly via the Zeeman torque induced by the magnetic field (3-5). Direct excitation has the advantage of minimal excess heat deposition but requires frequencies in the $10^{10}$ to $10^{12} \mathrm{~Hz}$ range. The low magnetic field strength of currently realizable THz-frequency EM sources poses a formidable challenge for such schemes.

Thanks to the coexistence of different ferroic orders, multiferroics offer new routes to domain control (6). Particularly strong coupling between the ferroelectric and magnetic order exists in single-phase frustrated magnets, where noncollinear spin structure drives ferroelectricity as a result of weak relativistic interactions (7-9). Consequently, the magnetic order can be controlled by application of an electric field (10-13).

${ }^{1}$ Institute for Quantum Electronics, Eidgenössische Technische Hochschule (ETH) Zürich, Wolfgang-Pauli-Strasse 16, 8093 Zürich, Switzerland. ${ }^{2}$ Swiss Light Source, Paul Scherrer Institut, 5232 Villigen PSI, Switzerland. ${ }^{3}$ Linac Coherent Light Source (LCLS), SLAC National Accelerator Laboratory, Menlo Park, CA94025, USA. ${ }^{4}$ SwissFEL, Paul Scherrer Institut, 5232 Villigen PSI, Switzerland. ${ }^{5}$ Advanced Light Source, Lawrence Berkeley National Laboratory, Berkeley, CA 94720, USA. ${ }^{6}$ Stanford Institute for Materials and Energy Sciences (SIMES), SLAC National Accelerator Laboratory, Menlo Park, CA 94025, USA. ${ }^{7}$ Ecole Polytechnique Federale de Lausanne, 1015 Lausanne, Switzerland. ${ }^{8}$ Institute for Quantum Matter (IQM), Department of Physics and Astronomy, Johns Hopkins University, Baltimore, MD 21218, USA.

*Corresponding author. E-mail: tkubacka@phys.ethz.ch †These authors contributed equally to this work. 